\title{
Crystallization and ferroelectric properties of the amorphous precursor films of Poly(vinylidene fluoride-trifluoroethylene) (P(VDF-TrFE)) blended with $\mathrm{Pb}(\mathrm{Zr}, \mathrm{Ti}) \mathrm{O}_{3}$
}

\author{
Wan-Gyu LEE, Byung Eun PARK ${ }^{*} \dagger$ and Kyung Eun PARK* \\ Department of Nano CMOS, National NanoFab Center, Daejeon 305-806, Korea \\ *School of Electrical and Computer Engineering, University of Seoul, Seoul 130-743, Korea
}

\begin{abstract}
Precursor films based on poly(vinylidene fluoride-trifluoroethylene) (P(VDF-TrFE)) and $\mathrm{P}(\mathrm{VDF}-\mathrm{TrFE})$ blended with $\mathrm{Pb}\left(\mathrm{Zr}\right.$,Ti) $\mathrm{O}_{3}$ (PZT) were spin-coated on Si substrates and subsequently annealed at 150,170 , or $190^{\circ} \mathrm{C}$. X-ray diffraction studies showed that the crystallization from the amorphous precursor films to the $\gamma$ phase starts at higher annealing temperatures without involving the formation of other polymorphs when the P(VDF-TrFE) is blended with PZT, and the PZT content increases, resulting in an amorphous phase and/or crystalline $\gamma$ phase. Nevertheless, a larger memory window width and much higher accumulation capacitance are induced by the blended PZT within the low operating voltage ranges from -0.5 to $2.0 \mathrm{~V}$ and from -2.0 to $6.0 \mathrm{~V}$ for 76.7 and 96.7 wt \% PZT blending, respectively. Furthermore, these improvements in the hysteretic characteristics in the capacitance-voltage measurements are also directly correlated with the degree of P(VDF-TrFE) crystallization and the presence of PZT. This approach enables viable routes toward the commercialization of nonvolatile ferroelectric memory devices and their market extension to potential applications as functional devices.
\end{abstract}

(๑2012 The Ceramic Society of Japan. All rights reserved.

Key-words : Ferroelectric, P(VDF-TrFE), PZT, Nonvolatile, Memory

[Received January 11, 2012; Accepted April 3, 2012]

Ferroelectric random access memory (FeRAM) is one of the nonvolatile memories now in production, and it has various attractive features (such as lower programming voltages, higher programming speeds, much longer endurance, and low power consumption) compared to its conventional counterparts such as Flash and EEPROM (Electrically Erasable Programming Read Only Memory). ${ }^{1,2)}$ Despite such high performance and commercialization, the process and materials for the production of FeRAM have not yet matured to support the coming ubiquitous technologies. Researchers have searched for appropriate materials for the realization of nonvolatile devices in both inorganic and organic fields.

Ferroelectric polymers have attracted continuous attention as functional devices for energy transduction and information storage or sensors. ${ }^{3)-5)}$ In particular, electronic devices based on organic materials have been widely investigated, opening a new path for the development of industrial devices with unique characteristics such as high flexibility, low weight, and low processing costs. Specifically, poly(vinylidene fluoride) (P(VDF)) and its copolymer with trifluoroethylene (P(VDF-TrFE)) are well known as ferroelectric polymers with large electric dipole moments, which are generated between the fluorine and hydrogen atoms in the individual vinylidene fluoride $\left(\mathrm{CH}_{2} \mathrm{CF}_{2} ; \mathrm{VDF}\right)$ units. ${ }^{6,7)}$ These polymers have four crystal phases with different lattice constants, molecular packing features, and conformations. They show different hysteretic characteristics depending on their crystal phases. For example, P(VDF) in the crystal phase (Form I) has large spontaneous polarizations because the electric dipoles are arranged in specific directions in the unit cell. For the realization of ferroelectric applications in $\mathrm{P}(\mathrm{VDF})$, it is necessary

\footnotetext{
Corresponding author: B. E. Park; E-mail: pbe@uos.ac.kr
}

to control the crystal structures. However, it is difficult to fabricate well-ordered polymer films with smooth surfaces and nanometer thicknesses, because polymers naturally have mixed structures of both crystalline and amorphous phases. The existence of the amorphous phase may be responsible for the decrease in ferroelectric performance. ${ }^{8)}$ In order to change the crystallization behavior of organic $\mathrm{P}(\mathrm{VDF}-\mathrm{TrFE})$ and thereby improve the ferroelectric properties of $\mathrm{P}(\mathrm{VDF}-\mathrm{TrFE})$, a commercial $\mathrm{Pb}(\mathrm{Zr}, \mathrm{Ti}) \mathrm{O}_{3}$ (PZT) precursor solution was blended with the $\mathrm{P}(\mathrm{VDF}-\mathrm{TrFE})$ solution. In addition, inorganic PZT has excellent ferroelectric properties, i.e., larger polarization and smaller coercive electric field than organic ferroelectric $\mathrm{P}(\mathrm{VDF}-\mathrm{TrFE})$. Thus, they are required for further integration of non-volatile RAM in next generation. This organic and inorganic combined product is expected to have the increased ferroelectric properties by the addition of PZT to the P(VDF-TrFE).

The ferroelectric organic material $\mathrm{P}(\mathrm{VDF}-\mathrm{TrFE})$ combined with the ferroelectric inorganic material PZT was designed to overcome the fundamental instabilities in the polar structure and to reduce the coercive field of $\mathrm{P}(\mathrm{VDF}-\mathrm{TrFE})$, which is larger than that of inorganic materials. We then investigated the phase formation and corresponding ferroelectric properties of an organic poly(vinylidene fluoride-trifluoroethylene (P(VDFTrFE)) thin film and of organic P(VDF-TrFE) thin films blended with inorganic amorphous PZT.

Amorphous precursor films were deposited from mixed solutions using a spin-coating process. One of the precursor solutions was prepared by dissolving $0.05 \mathrm{~g}$ of the vinylidene fluoridetrifluoroethylene P(VDF-TrFE) copolymer powder (99.9\%, SOLVAY) in $2.5 \mathrm{~mL}$ of dimethyl alcohol (99.9\%, Aldrich). The resulting solution had an atomic VDF/TrFE ratio of 1.08, which was a composition approximately midway between VDF and 


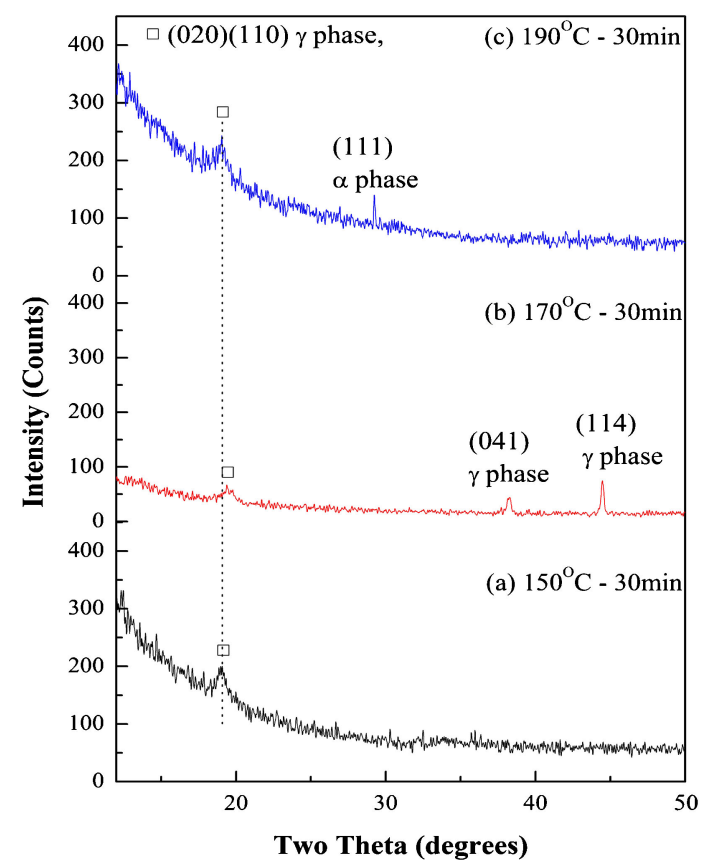

Fig. 1. (Color online) XRD patterns of the P(VDF-TrFE) films annealed at 150,170 , and $190^{\circ} \mathrm{C}$.

TrFE. The others were prepared by blending the P(VDF-TrFE) solution with different volumes of a $0.25 \mathrm{~mol}$ PZT solution (99.9\%, Inostek Inc.). The resulting precursor solutions had atomic $\mathrm{Pb} / \mathrm{Zr} / \mathrm{Ti}$ ratios of $110 / 35 / 65$, which is the ratio typically applied for FeRAM devices. The volume ratio between the $\mathrm{P}(\mathrm{VDF}-\mathrm{TrFE})$ and PZT solutions was 0.33:3.00. (100)-oriented $\mathrm{Si}$ single-crystal substrate was implanted with $\mathrm{BF}_{2}\left(4.0 \times 10^{15}\right.$ $\mathrm{cm}^{-2}$ at $\left.20 \mathrm{keV}\right)$ and followed by activation annealing $\left(1000^{\circ} \mathrm{C}\right.$ for $10 \mathrm{~s}$.), resulting in the sheet resistance of $58.5 \Omega / \square$. The substrates $(1 \mathrm{~cm} \times 1 \mathrm{~cm})$ doped with $B$ were coated with the precursor solutions at $1500 \mathrm{rpm}$ for $60 \mathrm{~s}$ under ambient conditions. The precursor films were subsequently pyrolyzed and annealed in a box furnace at 150,170 , and $190^{\circ} \mathrm{C}$ for $0.5 \mathrm{~h}$. The thicknesses were measured using an ellipsometer (M2000D, Woollam) after annealing the precursor films. X-ray diffraction (XRD) was used to assess the phases and crystallographic textures of the product films. Routine analysis was performed using a $2 \theta$ diffractometer (Rigaku, Tokyo, Japan) with monochromatized $\mathrm{Cu}-\mathrm{K}$ radiation that was supplied by a rotating anode generator operating at $40 \mathrm{kV}$ and $300 \mathrm{~mA}$ with a scanning speed of $2^{\circ} / \mathrm{min}$ and a step size of $0.01^{\circ}$. Au top electrodes were deposited on the formed ferroelectric films using electron-beam evaporation through a metal shadow mask. The capacitance versus voltage $(\mathrm{C}-\mathrm{V})$ characteristics shown in Figs. 4-6 were measured by sweeping the gate voltage from inversion to accumulation at room temperature.

X-ray diffraction patterns of the films annealed for $30 \mathrm{~min}$ at different temperatures (from 150 to $190^{\circ} \mathrm{C}$ ) are presented in Fig. 1. In Fig. 1(a), the crystalline phase was detected in the film that was annealed for $0.5 \mathrm{~h}$ at $150^{\circ} \mathrm{C}$. The pattern can be identified as belonging to the orthorhombic $\gamma$ phase (020) and/or (110) of $\mathrm{P}(\mathrm{VDF}-\mathrm{TrFE})$ at $2 \theta=19.1^{\circ}$. A shift in peak position was evident following the annealing at $170^{\circ} \mathrm{C}$, along with two sharp diffraction peaks at around $2 \theta=38.24^{\circ}$ and $44.44^{\circ}$. These peaks can be assigned to the (020), (110), (041), and (114) planes of the orthorhombic $\gamma$ phase of P(VDF-TrFE). Although annealing of the precursor film at $170^{\circ} \mathrm{C}$ resulted in the peak

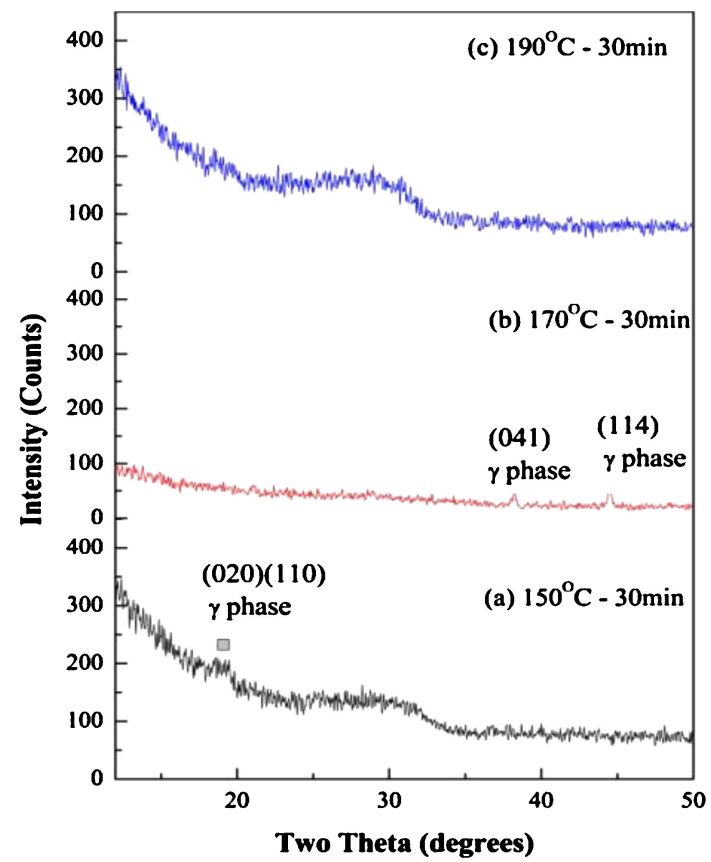

Fig. 2. (Color online) XRD patterns of the $76.7 \mathrm{wt} \%$ PZT blended $\mathrm{P}(\mathrm{VDF}-\mathrm{TrFE})$ films annealed at 150,170 , and $190^{\circ} \mathrm{C}$.

shifting closer to the (110) orthorhombic $\gamma$ phase, the peak still retained its double-peak character. An increase in the annealing temperature led to a shift of the peak position back from $2 \theta=$ $19.5^{\circ}$ to $2 \theta=19.1^{\circ}$, and to the appearance of an additional sharp diffraction peak at around $2 \theta=29.2^{\circ}$, which is due to the orthorhombic $\alpha$ phase (111). ${ }^{9}$ The XRD patterns obtained from the films annealed between 150 and $190^{\circ} \mathrm{C}$ all exhibited a common peak reflected at $2 \theta=19.1^{\circ}$ or $19.5^{\circ}$, whereas specific diffraction behavior was observed at each temperature.

Figure 2 shows the XRD patterns of the films blended with $76.7 \mathrm{wt} \%$ PZT and annealed at various temperatures. On annealing the precursor films of $\mathrm{P}(\mathrm{VDF}-\mathrm{TrFE})$ with $\mathrm{PZT}$ at $150^{\circ} \mathrm{C}$ for $0.5 \mathrm{~h}$, as shown in Fig. 2(a), there was a very interesting broadening of the annealing behavior of the precursor films. Significantly, all the peaks from the blended PZT films were suppressed, and the X-ray diffraction patterns resembled that of an amorphous film, except for that of the film blended with $76.7 \mathrm{wt} \% \mathrm{PZT}$ and annealed at $170^{\circ} \mathrm{C}$, in which there were two distinct, sharp peaks at $2 \theta=38.24$ and $44.44^{\circ}$, as shown in Fig. 2(b). The reason for this is probably the complete crystallization of the precursor film. In addition, this film did not exhibit the weak peak at $2 \theta=19.1$ or $19.5^{\circ}$, unlike the $\mathrm{P}(\mathrm{VDF}-\mathrm{TrFE})$ film annealed at the same temperature of $170^{\circ} \mathrm{C}$. With annealing at $190^{\circ} \mathrm{C}$, there was no evidence for crystallization of the film blended with $76.7 \mathrm{wt} \% \mathrm{PZT}$; this is attributable to the high annealing temperature close to the melting point and the rapid cooling down to room temperature. ${ }^{10)}$

Figure 3 shows the XRD patterns of the films blended with $96.7 \mathrm{wt} \%$ PZT and annealed at various temperatures. Further increasing the concentration to $96.7 \mathrm{wt} \%$ PZT suppressed the extent of crystallization more than for $76.7 \mathrm{wt} \% \mathrm{PZT}$, as shown by the disappearance of the weak peak at $2 \theta=19.1$ or $19.5^{\circ}$ for the film annealed at $150^{\circ} \mathrm{C}$, the reappearance of a broadening in the film annealed at $170^{\circ} \mathrm{C}$, and the strongest intensity of the amorphous phase in the film annealed at $190^{\circ} \mathrm{C}$. The increased thickness of the films should also be considered as a possible cause of the increased intensity. 


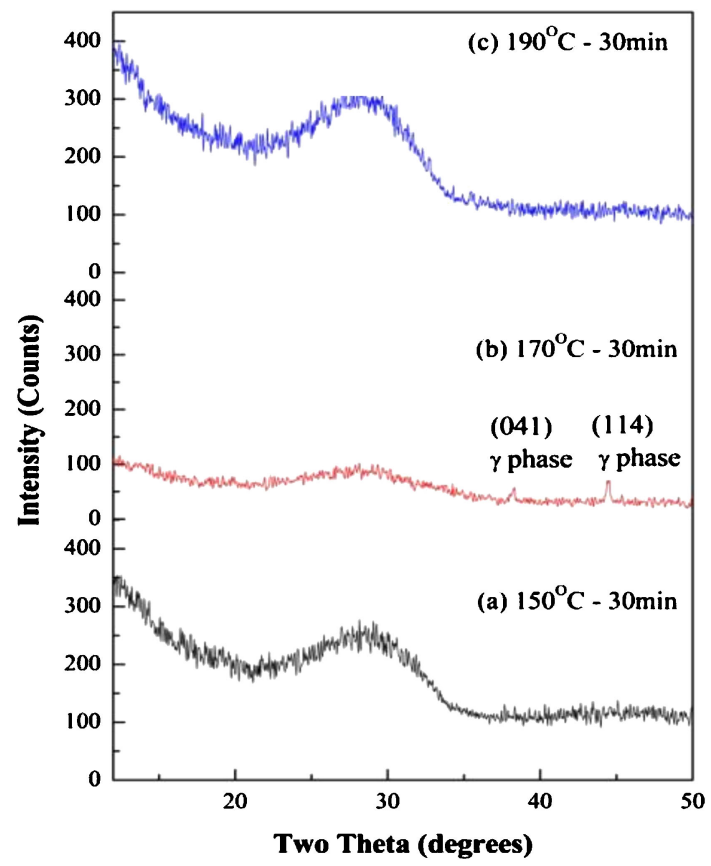

Fig. 3. (Color online) XRD patterns of the $96.7 \mathrm{wt} \%$ PZT blended $\mathrm{P}(\mathrm{VDF}-\mathrm{TrFE})$ films annealed at 150,170 , and $190^{\circ} \mathrm{C}$.

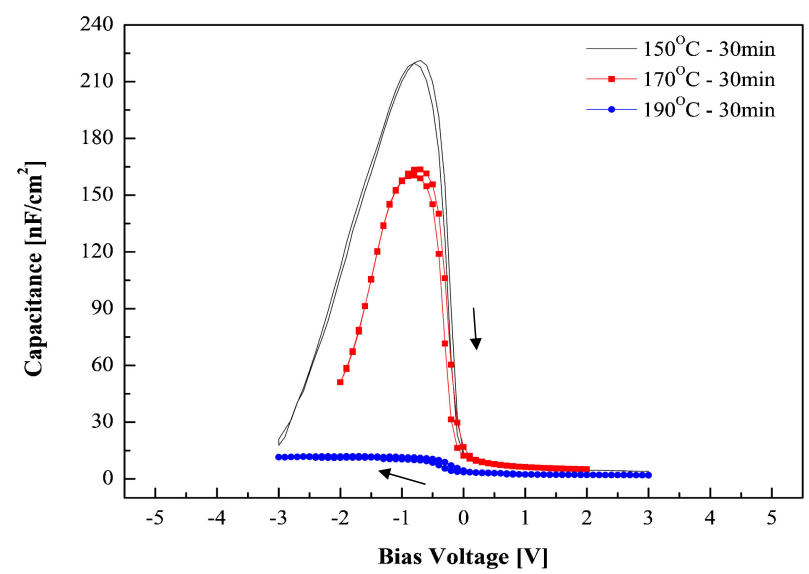

Fig. 4. (Color online) $\mathrm{C}-\mathrm{V}$ characteristics of $\mathrm{Au} / \mathrm{P}(\mathrm{VDF}-\mathrm{TrFE}) / \mathrm{Si}$ capacitors for different annealing temperatures of 150,170 , and $190^{\circ} \mathrm{C}$. These correspond to the results shown in Fig. 1.

Figure 4 shows the typical $\mathrm{C}-\mathrm{V}$ curves of $\mathrm{Au} / \mathrm{P}(\mathrm{VDF}-\mathrm{TrFE}) /$ $\mathrm{Si}$-substrate capacitors annealed at different temperatures. Clockwise hysteretic behavior was observed in the P(VDF-TrFE) films with a narrow memory window. The capacitance of the P(VDFTrFE) films decreased with increasing annealing temperature. The different values of accumulation capacitance originated mainly from the crystalline polymorphs and partly from the different vector sums of the constituent dipoles. On the basis of the preceding XRD results (as shown in Fig. 1), one can conclude that the mixture of the $\gamma$ and $\alpha$ phases reduces the accumulation capacitance much more than the mixture of the preferred orientations of the $\gamma$ phase, since among the four crystalline polymorphs, only the $\alpha$ phase is a non-polar phase. The $\beta$ and $\delta$ phases were not formed by annealing the precursor $\mathrm{P}(\mathrm{VDF}-\mathrm{TrFE})$ films.

Figure 5 shows the typical $\mathrm{C}-\mathrm{V}$ curves of $\mathrm{Au} / 76.7 \mathrm{wt} \%$ PZTblended $\mathrm{P}(\mathrm{VDF}-\mathrm{TrFE})$ films/Si-substrate capacitors annealed at

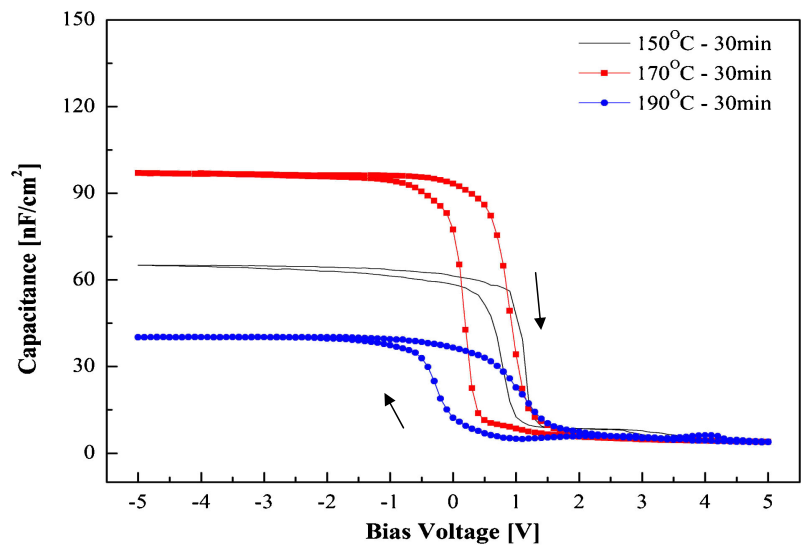

Fig. 5. (Color online) $\mathrm{C}-\mathrm{V}$ characteristics of $\mathrm{Au} / \mathrm{P}(\mathrm{VDF}-\mathrm{TrFE})$ blended with $76.7 \mathrm{wt} \% \mathrm{PZT} / \mathrm{Si}$ capacitors for different annealing temperatures of 150,170 , and $190^{\circ} \mathrm{C}$. These correspond to the results shown in Fig. 2.

different temperatures. The capacitance versus voltage curves ( $\mathrm{C}-\mathrm{V}$ characteristics) were enhanced by blending $\mathrm{Pb}(\mathrm{Zr}, \mathrm{Ti}) \mathrm{O}_{3}$, as shown in Fig. 5. These improvements included: (1) a stronger polarization effect than in the $\mathrm{P}(\mathrm{VDF}-\mathrm{TrFE})$ films; (2) an increase in memory window width; and (3) a lower operating voltage. As pointed out earlier, ${ }^{8)}$ ferroelectric behavior stems primarily from the crystallization but is more or less from the amorphous phase. Furthermore, it can also be seen in Fig. 5 that the ferroelectric property corresponding to the accumulation capacitance is mainly determined by the degree of crystallization, or volume fraction of crystallization. For example, the accumulated capacitance is about $37.4,61.4$, and $94.4 \mathrm{nF} / \mathrm{cm}^{2}$ at $-1 \mathrm{~V}$, corresponding to no crystallization (blue curve in Fig. 5), partial crystallization (black curve in Fig. 5), and complete crystallization (red curve in Fig. 5), respectively. However, the memory window width does not show the same trend as the accumulated capacitance, which suggests that the memory window width is not a function of the degree of $\mathrm{P}(\mathrm{VDF}-\mathrm{TrFE})$ crystallization but of the combined state of P(VDF-TrFE) and PZT depending upon the annealing temperature.

Figure 6 shows more improvements in the memory window width for the $96.7 \mathrm{wt} \%$ PZT-blended P(VDF-TrFE) films. The memory window width of the films are strongly dependent on the annealing temperature, similarly to the results shown in Fig. 5. With annealing at $150^{\circ} \mathrm{C}$, the memory window width is about $1.4 \mathrm{~V}$, which increases further to 1.6 and $3.2 \mathrm{~V}$ on annealing at 170 and $190^{\circ} \mathrm{C}$, respectively. Compared to the results for the $\mathrm{P}(\mathrm{VDF}-\mathrm{TrFE})$ films and $\mathrm{P}(\mathrm{VDF}-\mathrm{TrFE})$ blended with $76.7 \mathrm{wt} \%$ PZT films annealed at the same temperature, these values are relatively high. It is therefore noticed that the addition of PZT increases ferroelectric property corresponding to memory window width while it rather suppresses the crystallization of $\mathrm{P}(\mathrm{VDF}-\mathrm{TrFE})$ determining the accumulation capacitance in ferroelectric properties. The increased capacitance of the 96.7 wt $\%$ PZT-blended film annealed at $170^{\circ} \mathrm{C}$ is mainly due to the partial crystallization of the $\gamma$ phase, as observed by XRD and described in Fig. 3. The crystallization of the precursor films with higher PZT contents starts at a higher annealing temperature of $170^{\circ} \mathrm{C}$ with different preferred orientations, confirming again that the blended PZT suppresses the crystallization of the amorphous precursor, and thus suggesting that the increase in PZT concentration is accompanied by an increase in crystallization temperature. 


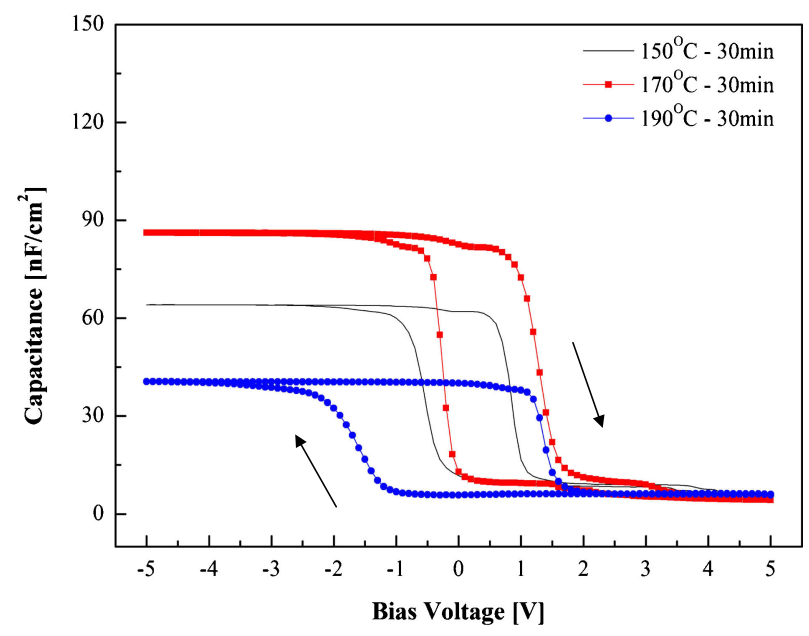

Fig. 6. (Color online) $\mathrm{C}-\mathrm{V}$ characteristics of Au/P(VDF-TrFE) blended with $96.7 \mathrm{wt} \% \mathrm{PZT} / \mathrm{Si}$ capacitors for different annealing temperatures of 150,170 , and $190^{\circ} \mathrm{C}$. These correspond to the results shown in Fig. 3.

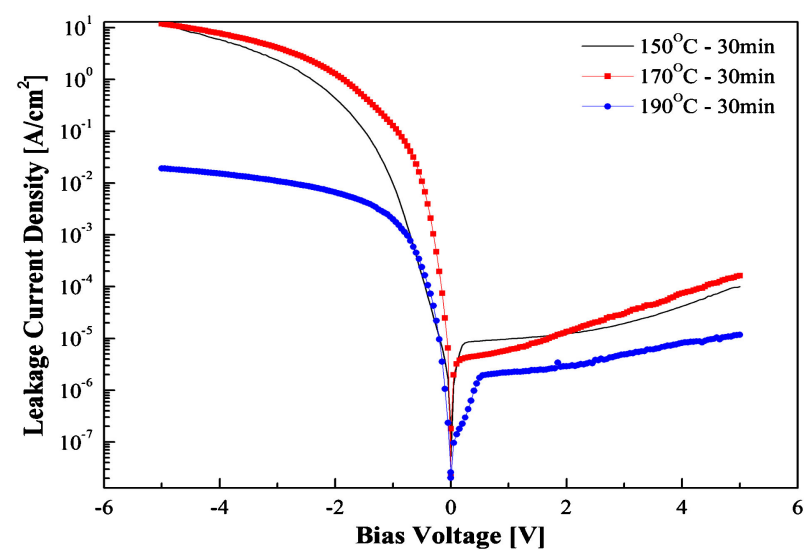

Fig. 7. (Color online) J-V characteristics of the same Au/P(VDF$\mathrm{TrFE}) / \mathrm{Si}$ capacitors as those in Fig. 4., for different annealing temperatures of 150,170 , and $190^{\circ} \mathrm{C}$.

When the film is annealed at $190^{\circ} \mathrm{C}$, no crystallization occurs. As a result, the accumulated capacitance decreases to $40 \mathrm{nF} / \mathrm{cm}^{2}$, which is lower than the value of $70 \mathrm{nF} / \mathrm{cm}^{2}$ for the film annealed at $150^{\circ} \mathrm{C}$. Although the two films annealed at 150 and $190^{\circ} \mathrm{C}$ have the same amorphous phase, they show different $\mathrm{C}-\mathrm{V}$ behaviors. On the basis of the XRD results in Fig. 1(c), it is thought that the $\alpha$ phase was formed by a two-step process: melting at the annealing temperature of $190^{\circ} \mathrm{C}$ in the first step, and crystallization during the rapid cooling to room temperature in the second step. This phase is termed the melt-crystallized $\alpha$ phase. ${ }^{10)}$ However, crystallization from the melt of PZT-containing precursor films to a solid in $\alpha$ phase is very difficult because of the PZT effect of suppressing the crystallization. In addition, from the viewpoint of ferroelectric properties, melting of the ferroelectric material takes the polarized properties toward the nonpolar ones. These facts thus explain the lower value of the accumulated capacitance of the film after annealing at $190^{\circ} \mathrm{C}$.

The leakage current density versus bias voltage $(\mathrm{J}-\mathrm{V})$ characteristics for the $\mathrm{Au} / \mathrm{P}(\mathrm{VDF}-\mathrm{TrFE}) / \mathrm{Si}$-substrates capacitors is shown in Fig. 7. The leakage current of $10.5 \mathrm{~nm}$ P(VDF-TrFE) films at a bias voltage of $-1.0 \mathrm{~V}$ were approximately $1.0 \times 10^{-2}$, $0.5 \times 10^{-1}$, and $2.0 \times 10^{-3} \mathrm{~A} / \mathrm{cm}^{2}$ for the annealing temper-

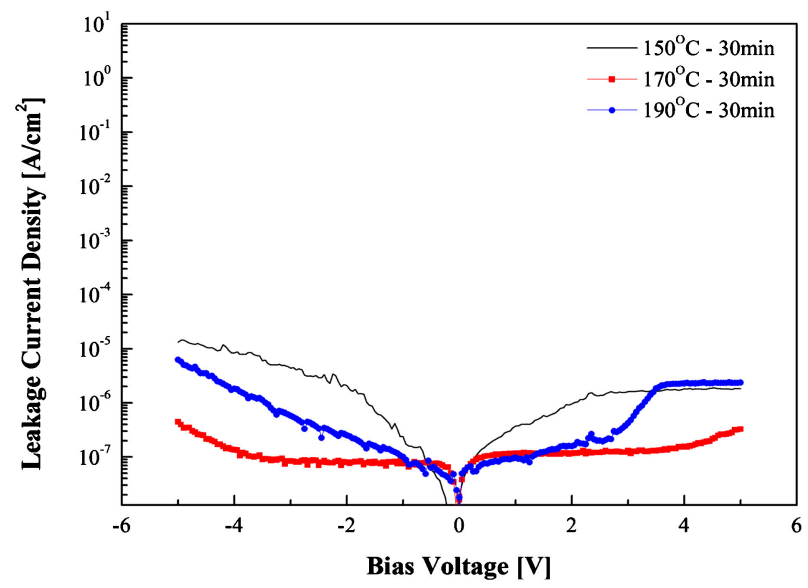

Fig. 8. (Color online) J-V characteristics of the same Au/P(VDFTrFE) blended with $76.7 \mathrm{wt} \% \mathrm{PZT} / \mathrm{Si}$ capacitors as those in Fig. 5., for different annealing temperatures of 150,170 , and $190^{\circ} \mathrm{C}$.

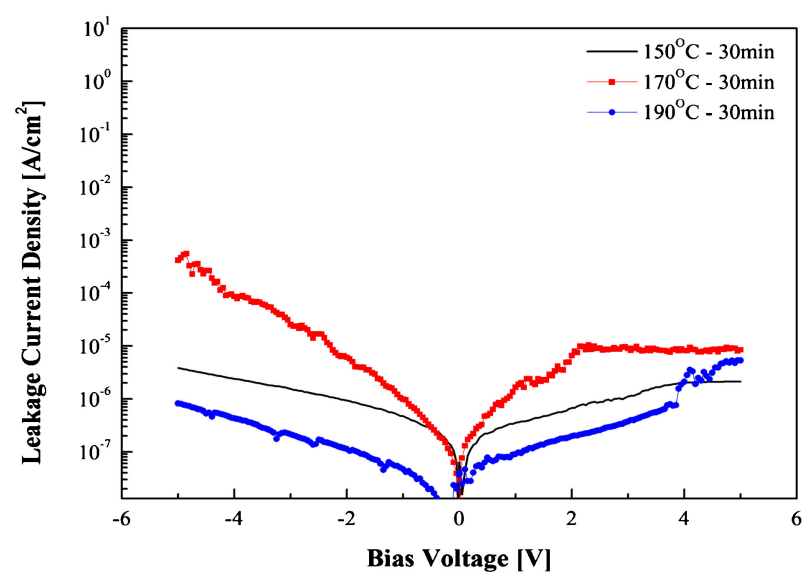

Fig. 9. (Color online) J-V characteristics of the same Au/P(VDFTrFE) blended with $96.7 \mathrm{wt} \% \mathrm{PZT} / \mathrm{Si}$ capacitors as those in Fig. 6., for different annealing temperatures of 150,170 , and $190^{\circ} \mathrm{C}$.

Table 1. Thicknesses of the P(VDF-TrFE) films blended with PZT and a $\mathrm{P}(\mathrm{VDF}-\mathrm{TrFE})$ film annealed for $0.5 \mathrm{~h}$ in air

\begin{tabular}{cc}
\hline Films & Thickness (nm) \\
\hline P(VDF-TrFE) & 10.5 \\
P(VDF-TrFE) with 76.7 wt \% PZT & 36.8 \\
P(VDF-TrFE) with 96.7 wt \% PZT & 117.0 \\
\hline
\end{tabular}

atures of 150,170 , and $190^{\circ} \mathrm{C}$, respectively. Based on the results of Fig. 1(c), it is clear that the lowering of leakage current level and of strong voltage dependence is attributed to the formation of $\alpha$ phase that is a non-polar phase. However, the mixture of preferred orientations, as shown in Fig. 1(b), aggravates the leakage current of $10.5 \mathrm{~nm}$ P(VDF-TrFE) film. The leakage current density of the films containing $76.7,96.7 \mathrm{wt} \% \mathrm{PZT}$ is relatively low in comparison with those of P(VDF-TrFE) films, as shown in Figs. 8 and 9. These are mainly caused by the film thickness and partly by the formed phases. The thicknesses of the films are summarized in Table 1. The phase mixture of $\gamma$ phase and amorphous phase leads to the highest leakage current density in both the 76.7 and $96.7 \mathrm{wt} \%$ PZT blended films, corresponding to the XRD result of Fig. 2(a) and of Fig. 3(b). However, single phase, what phase it maybe an amorphous or $\gamma$ phase, lowers the 
leakage current density in both the 76.7 and $96.7 \mathrm{wt} \%$ PZT blended films. Although the same amorphous phase is formed for the $96.7 \mathrm{wt} \%$ PZT blended films that were annealed at 150 and $190^{\circ} \mathrm{C}$, higher-temperature annealing provides better leakage current property than lower-temperature annealing. For the 76.7 wt \% PZT blended films, the lowest level of leakage current density at $-1.0 \mathrm{~V}$ is approximately $1 \times 10^{-7} \mathrm{~A} / \mathrm{cm}^{2}$ which is comparable to the results of inorganic strontium bismuth tantalite (SBT) films on an $\mathrm{HfO}_{2} \cdot{ }^{11)}$ Furthermore, it shows the bias voltage independence from $-4.0 \mathrm{~V}$ to $+4.0 \mathrm{~V}$ with a symmetric feature of leakage current density versus bias voltage. Considering simultaneously film thickness, leakage current density, and ferroelectric properties of the films, the $76.7 \mathrm{wt} \%$ PZT blended films are better than other films.

In summary, amorphous and/or crystalline thin films of P(VDF-TrFE) blended with 76.7 and $96.7 \mathrm{wt} \%$ PZT on $\mathrm{Si}$ (100) were formed at annealing temperatures of 150,170 , and $190^{\circ} \mathrm{C}$, and their crystallization and ferroelectric properties were characterized using XRD and $\mathrm{C}-\mathrm{V}$ analyses. The X-ray diffraction data showed that the precursor films crystallize to the $\gamma$ phase at higher annealing temperatures when they are blended with PZT, without involving the formation of polymorphs. The crystallization temperature increases as the PZT content increases. The improvement in the hysteretic $\mathrm{C}-\mathrm{V}$ characteristics is directly correlated with the degree of crystallization. Furthermore, the blended PZT induces a much larger memory window width and higher accumulation capacitance within the low operating voltage range from -0.5 to $2.0 \mathrm{~V}$ for the $76.7 \mathrm{wt} \%$ PZT blending, and from -2.0 to $6.0 \mathrm{~V}$ for the $96.7 \mathrm{wt} \% \mathrm{PZT}$ blending, even if the formed phase is amorphous or the $\gamma$ phase. Compared to the P(VDF-TrFE) films, the bias voltage independence of leakage current and reduction in the much lower level of leakage current, $10^{-7}$ were obtained at approximately $0.27 \mathrm{MV} /$ $\mathrm{cm}$ in the $76.7 \mathrm{wt} \%$ PZT blended films when the formed phases were not phase mixtures but a single $\gamma$ phase. The low-voltage operation, low leakage current, and high performance capacitance with the $\gamma$ phase lead to new ways to extend the commercial market of nonvolatile high-density FeRAM devices and their potential applications as functional devices.

Acknowledgement This research was supported by Basic Science Research Program through the National Research Foundation of Korea (NRF) funded by the Ministry of Education, Science and Technology (2011-0026604).

\section{References}

1) J. F. Scott and C. A. Paz de Araujo, Science, 246, 1400-1405 (1989).

2) C. A. Pas De Araujo, J. D. Cuchiaro, L. D. McMillan, M. C. Scott and J. F. Scott, Nature, 374, 627-629 (1995).

3) S. Fujisaki, H. Ishiwara and Y. Fujisaki, Appl. Phys. Express., 1, 081801 (2008).

4) R. C. G. Naber, K. Asadi, P. W. M. Blom, D. M. de Leeuw and B. de Boer, Adv. Mater., 22, 933-945 (2010).

5) Q. M. Zhang, H. Li, M. Poh, F. Xia, Z.-Y. Cheng, H. Xu and C. Huang, Nature, 419, 284-287 (2002).

6) H. Kawai, Jpn. J. Appl. Phys., 8, 975-976 (1969).

7) J. G. Bergman, Jr., J. H. McFee and G. R. Crane, Appl. Phys. Lett., 18, 203-205 (1971).

8) T. Furukawa, Phase Transit., 18, 143-211 (1989).

9) A. J. Lovinger and T. T. Wang, Polymer, 20, 725-732 (1979).

10) S. Weinhold, M. H. Litt and J. B. Lando, Macromolecules, 13, 1178-1183 (1980).

11) B.-E. Park, K. Takahashi and H. Ishiwara, Appl. Phys. Lett., $85,4448-4450$ (2004). 